\title{
Erratum: Giant photonic spin Hall effect near the Dirac points [Phys. Rev. A 101, 023826 (2020)]
}

\author{
Wenhao Xu, Qiang Yang, Guangzhou Ye, Weijie Wu, Wenshuai Zhang, Hailu Luo @e, and Shuangchun Wen
}

(Received 2 June 2020; published 19 June 2020)

DOI: 10.1103/PhysRevA.101.069903

Due to an error in the discussion of the arbitrary wave vector of the Gaussian beam, we introduced optical axis direction $\mathcal{I}=\alpha \hat{\mathbf{x}}+\beta \hat{\mathbf{y}}+\gamma \hat{\mathbf{z}}$ to describe components of the arbitrary vector under rotating transition. But the assumption of $\gamma \neq 0$ does not strictly conform boundary conditions at the interface in this paper. This was incorrect in the earlier implementation. So, we reset the optical axis on the $x y$ plane $(\gamma=0)$ and almost along the $x$ axis, and $\beta$ can be indicated with the arbitrary wave-vector $\beta \sim \frac{k_{i y}}{k_{i} \sin \theta_{i}}$ in a slight angle rotation.

The correction forms of Fresnel's coefficients are obtained by using the paraxial approximation, i.e., higher-order terms of the arbitrary vector have been neglected, and only the first order is retained. Our Eqs. (6)-(9) in the original paper should, therefore, be given, instead, by the following expressions:

$$
\begin{gathered}
r_{p p}=\frac{-q_{e}+\varepsilon_{x} \cos \theta_{i}}{q_{e}+\varepsilon_{x} \cos \theta_{i}}, \\
r_{s s}=\frac{-q_{o}+\mu_{x} \cos \theta_{i}}{q_{o}+\mu_{x} \cos \theta_{i}}, \\
r_{p s}=\frac{2 \frac{k_{i y}}{k_{i} \sin \theta_{i}} \sqrt{\varepsilon_{x} \mu_{x}}\left(\sqrt{\varepsilon_{x} \mu_{x}}-\sqrt{\varepsilon_{y} \mu_{y}}\right) \cos \theta_{i}}{\left(q_{e}+\varepsilon_{x} \cos \theta_{i}\right)\left(q_{o}+\mu_{x} \cos \theta_{i}\right)}, \\
r_{s p}=\frac{2 \frac{k_{i y}}{k_{i} \sin \theta_{i}} \sqrt{\varepsilon_{x} \mu_{x}}\left(\sqrt{\varepsilon_{y} \mu_{y}}-\sqrt{\varepsilon_{x} \mu_{x}}\right) \cos \theta_{i}}{\left(q_{e}+\varepsilon_{x} \cos \theta_{i}\right)\left(q_{o}+\mu_{x} \cos \theta_{i}\right)} .
\end{gathered}
$$

To obtain a precise model of the Gaussian beam, we introduce Taylor series expansion based on the arbitrary angular spectrum component, and the reflection coefficients are expanded as a polynomial of $k_{i x}$,

$$
r_{A}\left(k_{i x}\right)=r_{A}\left(k_{i x}=0\right)+k_{i x}\left[\frac{\partial r_{A}\left(k_{i x}\right)}{\partial k_{i x}}\right]_{k_{i x}=0}+\sum_{j=2}^{N} \frac{k_{i x}^{j}}{j !}\left[\frac{\partial^{j} r_{A}\left(k_{i x}\right)}{\partial k_{i x}^{j}}\right]_{k_{i x}=0},
$$

where $A \in\{p p, s s, p s, s p\}$. Here, we also retained the reflection coefficients to the first order.

The correction has no consequences on any of the discussed effects, i.e., when the Gaussian beam impinges on the unique interface, we found that the strong photonic spin Hall effect (SHE) near the Dirac point manifests itself as a large spin-dependent splitting in position spaces. The giant photonic SHE is attributed to the strong spin-orbit interaction of light, which manifests itself as the large polarization rotations of different plane-wave components. 